\title{
Modeling and Analysis of the Dynamic Behavior for Interacting Cracks on Circular Cavities in Piezoelectric Material
}

\author{
Dong $\mathrm{LI}^{1, \mathrm{a},{ }^{*}, \text { Hui-Cong WANG }}{ }^{1, \mathrm{~b}}$ \\ ${ }^{1}$ Department of Civil Engineering, Hebei Jiaotong Vocational \& Technical College, \\ Shijiazhuang050091, PR China \\ alidong242@163.com, bwanghcong@126.com \\ ${ }^{*}$ Corresponding author
}

Keywords: Radial Crack, Piezoelectric Material, Green's Function, Dynamic Stress Intensity Factor (DSIF), SH-wave.

\begin{abstract}
The present paper is exposed theoretically to the influence on the dynamic stress intensity factor (DSIF) in the piezoelectric material model with two permeable radial cracks emanating from the edges of two circular cavities, subjected to the dynamic incident anti-plane shearing wave (SH-wave). Green's functions are established based on complex variable and wave function expansion methods. Conjunction and crack-simulation techniques are used to evaluate DSIFs at the cracks' tip. The problem is reduced to a series of Fredholm integral equations of first kind. The numerical results are obtained by solving the equations to show the influence of the structural geometry, and the wave frequencies of incident wave on the dimensionless DSIFs.
\end{abstract}

\section{Introduction}

In modern science technology, important smart materials as piezoelectric materials are utilized widely due to their electro-mechanical coupling response. For instance, the piezoelectric materials have been used to establish many devices in the health monitoring of engineering structures as sensors, actuators, etc. Occasionally, because the materials stiff and brittle nature, newly developed piezoelectric devices acquire fracture or failure during manufacturing, polling process and service procedures. Therefore, researchers paid great attention to the failure behaviors and influences caused by defects in those devices.

Significant efforts had been made to the study of the electro-mechanical behavior of piezoelectric materials. For example, Wang[1-2] examined theoretically the dynamic behavior of piezoelectric materials containing interacting cracks under anti-plane mechanical and in-plane electric loading. By Green's function, Liu and Chen[3] studied cases by radial cracks of any limited length along the radius originating at an elliptical hole. Guo et al.[4] analyzed the problem of two non-symmetrical collinear cracks emanating from an elliptical hole in a piezoelectric solid. Hassan and Song[5] studied the dynamic stress intensity factor in the piezoelectric bi-materials model with two symmetrically permeable interfacial cracks near the edges of a circular cavity.

In the present paper, the dynamic stress intensity factors (DSIFs) in transversely isotropic piezoelectric material, which contain radial cracks emanating from the circular cavities, are studied under the incident anti-plane shearing wave. The emphasis is placed on the dynamic stress intensity factors at the tips of the cracks based on complex variable and Green's function methods.

\section{Fundamental Equations}

The geometries of the analysis model are shown in Fig. 1, where two radial cracks emanating from the edge of the circular cavities at transversely isotropic piezoelectric materials in the $x-y$ plane (or $r$ - $\theta$ plane). The poling direction is the positive $z$-axis. $A_{1}$ and $A_{2}$ represent the cracks' lengths, and $d$ represents the distance between the crack's tips. $R_{1}$ and $R_{2}$ are the radii of the circular cavities. The model is subjected to a dynamic SH-wave, whose incident angle is $\alpha_{0}$. 


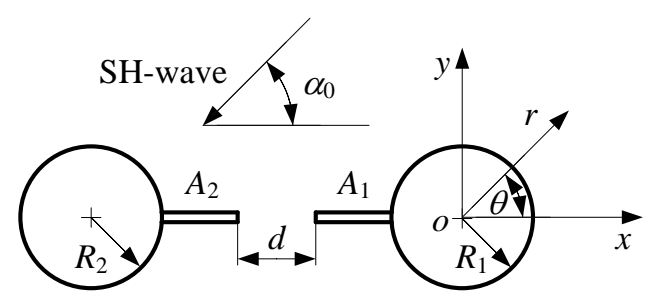

Fig. 1 Piezoelectric material with radial cracks emanating from the edges of circular cavities

The governing equations of linear piezoelectricity can be specified as:

$$
c_{44} \nabla^{2} w+e_{15} \nabla^{2} \phi+\rho \omega^{2} w=0, e_{15} \nabla^{2} w-\kappa_{11} \nabla^{2} \phi=0,
$$

in which, $c_{44}, e_{15}$ and $\kappa_{11}$ are shear elastic modulus, piezoelectric constant and dielectric constant of the medium, respectively; while $w, \phi, \rho$ and $\omega$ are anti-plane displacement, electric potential, mass density and frequency of the incident wave, respectively.

By introducing a complex variable $\eta=x+i y=r e^{i \theta}$ and its conjugate $\bar{\eta}=x-i y=r e^{-i \theta}$, the Eq. 1 can be simplified further as:

$$
\frac{\partial^{2} w}{\partial \eta \partial \bar{\eta}}+\frac{1}{4} k^{2} w=0, \frac{\partial^{2} \varphi}{\partial \eta \partial \bar{\eta}}=0 \quad \phi=\frac{e_{15}}{\kappa_{11}} w+\varphi
$$

where $k^{2}=\rho \omega^{2} / c^{*}, c^{*}=c_{44}(1+\lambda), \lambda=e_{15}^{2} / c_{44} \kappa_{11}$.

The constitutive relations for the piezoelectric material can be expressed by

$$
\begin{aligned}
& \tau_{r z}=c_{44}\left(\frac{\partial w}{\partial \eta} e^{i \theta}+\frac{\partial w}{\partial \bar{\eta}} e^{-i \theta}\right)+e_{15}\left(\frac{\partial \phi}{\partial \eta} e^{i \theta}+\frac{\partial \phi}{\partial \bar{\eta}} e^{-i \theta}\right), \tau_{\theta z}=i c_{44}\left(\frac{\partial w}{\partial \eta} e^{i \theta}-\frac{\partial w}{\partial \bar{\eta}} e^{-i \theta}\right)+i e_{15}\left(\frac{\partial \phi}{\partial \eta} e^{i \theta}-\frac{\partial \phi}{\partial \bar{\eta}} e^{-i \theta}\right) \\
& D_{r}=e_{15}\left(\frac{\partial w}{\partial \eta} e^{i \theta}+\frac{\partial w}{\partial \bar{\eta}} e^{-i \theta}\right)-\kappa_{11}\left(\frac{\partial \phi}{\partial \eta} e^{i \theta}+\frac{\partial \phi}{\partial \bar{\eta}} e^{-i \theta}\right), D_{\theta}=i e_{15}\left(\frac{\partial w}{\partial \eta} e^{i \theta}-\frac{\partial w}{\partial \bar{\eta}} e^{-i \theta}\right)-i \kappa_{11}\left(\frac{\partial \phi}{\partial \eta} e^{i \theta}-\frac{\partial \phi}{\partial \bar{\eta}} e^{-i \theta}\right)
\end{aligned}
$$

where $\tau_{r z}, \tau_{\theta z}, D_{r}$ and $D_{\theta}$ are two shear stress components and two electric displacement components, respectively.

\section{Boundary Value Problem and DSIF}

Firstly, construct a couple of Green's functions. The Green's functions of elastic displacement $G_{w}$ and electric potential $G_{\phi}$ for a semi-infinite piezoelectric medium with two circular cavities impacted by an out-plane harmonic line source loading $\delta\left(\eta-\eta_{0}\right)$ at the horizontal surface can be described as:

$$
\begin{aligned}
& G_{w}=\frac{i}{2 c^{*}} H_{0}^{(1)}\left(k\left|\eta-\eta_{0}\right|\right)+\sum_{j=1}^{2} \sum_{m=-\infty}^{\infty} A_{m}^{j} H_{m}^{(1)}\left(k\left|\eta_{j}\right|\right) \cdot\left[\left(\frac{\eta_{j}}{\left|\eta_{j}\right|}\right)^{m}+\left(\frac{\eta_{j}}{\left|\eta_{j}\right|}\right)^{-m}\right], \\
& G_{\phi}=\frac{e_{15}}{\kappa_{11}} G_{w}+\sum_{j=1}^{2} \sum_{m=1}^{\infty}\left[B_{m}^{j} \eta_{j}^{-m}+C_{m}^{j} \bar{\eta}_{j}^{-m}\right]
\end{aligned}
$$


And the electric potential in the circular cavities can be written as:

$$
G_{\phi}^{\mathrm{Ij}}=D_{0}+\sum_{m=1}^{\infty}\left[D_{m}^{j} \eta_{j}^{m}+E_{m}^{j} \bar{\eta}_{j}^{m}\right]
$$

The unknown coefficients can be obtained by the boundary conditions as following

$$
\tau_{r z}^{j}=0, \quad D_{r}=D_{r}^{\mathrm{Ij}}, \quad G_{\phi}=G_{\phi}^{\mathrm{Ij}}, \quad\left|\eta_{j}\right|=R_{j} \quad(j=1,2) .
$$

Secondly, Consider a piezoelectric material with two circular cavities subjected to the dynamic incident SH-wave directed with an angle $\alpha_{0}$. The incident elastic displacement and electric potential fields in piezoelectric material are:

$$
w^{(i)}=w_{0} \cdot \exp \left\{-i \frac{k}{2}\left[(\eta+i h) e^{-i \alpha_{0}}+(\bar{\eta}-i h) e^{i \alpha_{0}}\right]\right\}, \quad \phi^{(i)}=\frac{e_{15}}{\kappa_{11}} w^{(i)} .
$$

The scattering fields by two circular cavities can be given as:

$$
w^{(s)}=\sum_{j=1}^{2} \sum_{m=-\infty}^{\infty}{ }^{j} A_{m}^{s} H_{m}^{(1)}\left(k\left|\eta_{j}\right|\right)\left(\frac{\eta_{j}}{\left|\eta_{j}\right|}\right)^{m}, \quad \phi^{(s)}=\frac{e_{15}}{\kappa_{11}} w^{(s)}+\sum_{j=1}^{2} \sum_{m=1}^{+\infty}{ }^{j} B_{m}^{s} \eta_{j}^{-m}+\sum_{j=1}^{2} \sum_{m=1}^{+\infty}{ }^{j} C_{m}^{s} \bar{\eta}_{j}^{-m}
$$

The electric potential inside the cavities can be written as:

$$
\phi_{j}^{c}={ }^{j} D_{0}^{s}+\sum_{m=1}^{\infty}\left[{ }^{j} D_{m}^{s} \eta_{j}^{m}+{ }^{j} E_{m}^{s} \bar{\eta}^{m}\right]
$$

The values of the coefficients can be obtained by the boundary conditions in Eq. 7 .

Thirdly, the integral equations can be established by the conjunction and crack-simulation technique. The infinite piezoelectric material is separated at $y=0$, bearing in mind the absence of the cracks. To overcome this and achieve the assumption of traction free and electric permeable cracks, a pair of negative shear stresses $-\tau_{\theta z}^{+}$and $-\tau_{\theta z}^{-}$are applied on the surfaces at the cracks' locations as shown in Fig. 2. So as to meet the continuity conditions of elastic displacements, a pair of additional shear stresses $f^{+}\left(r_{0}, \theta_{0}\right), f^{-}\left(r_{0}, \theta_{0}\right)$ are applied on the surfaces out of the cracks. The integral equations to solve the unknown stress can be obtained according to the continuity conditions at the surface.

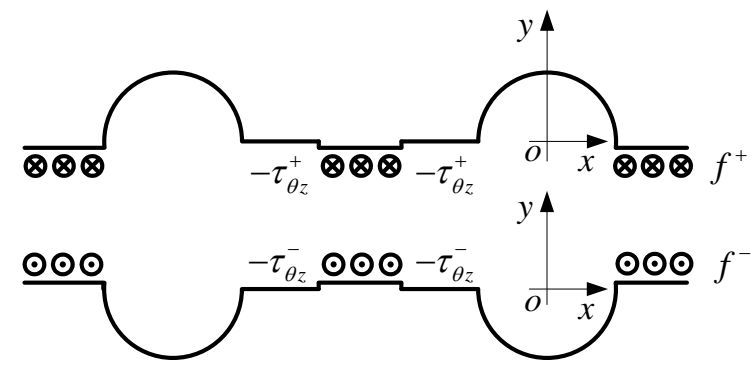

Fig. 2 Conjunction for piezoelectric material with four semi-circular notches

The continuity conditions of the shear stresses at the surfaces out of the cracks can be written as: 
$\tau_{\theta z}^{+} \cos \theta_{0}+f^{+}\left(r_{0}, \theta_{0}\right)=\tau_{\theta z}^{-} \cos \theta_{0}+f^{-}\left(r_{0}, \theta_{0}\right)$

The continuity conditions of elastic displacements at the surface can be expressed as:

$$
w^{(t+)}+w^{(f+)}+w^{(c+)}=w^{(t-)}+w^{(f-)}+w^{(c-)}
$$

in which, $\tau_{\theta z}^{+}=\tau_{\theta z}^{-}=\tau_{\theta z}^{(t)}=\tau_{\theta z}^{(i)}+\tau_{\theta z}^{(s)}, w^{(t+)}=w^{(t-)}=w^{(t)}=w^{(i)}+w^{(s)}$,

$$
\begin{aligned}
& w^{(f+)}=\int_{\Gamma_{1}} f^{+}\left(r_{0}, \pi\right) G_{w}\left(r, \theta ; r_{0}, \pi\right) d r_{0}+\int_{\Gamma_{2}} f^{+}\left(r_{0}, \pi\right) G_{w}\left(r, \theta ; r_{0}, \pi\right) d r_{0}+\int_{\Gamma_{3}} f^{+}\left(r_{0}, 0\right) G_{w}\left(r, \theta ; r_{0}, 0\right) d r_{0}, \\
& w^{(f-)}=-\int_{\Gamma_{1}} f^{-}\left(r_{0}, \pi\right) G_{w}\left(r, \theta ; r_{0}, \pi\right) d r_{0}-\int_{\Gamma_{2}} f^{-}\left(r_{0}, \pi\right) G_{w}\left(r, \theta ; r_{0}, \pi\right) d r_{0}-\int_{\Gamma_{3}} f^{-}\left(r_{0}, 0\right) G_{w}\left(r, \theta ; r_{0}, 0\right) d r_{0}, \\
& w^{(c+)}=\int_{\Pi_{1}} \tau_{\theta z}^{(t)}\left(r_{0}, \pi\right) G_{w}\left(r, \theta ; r_{0}, \pi\right) d r_{0}+\int_{\Pi_{2}} \tau_{\theta z}^{(t)}\left(r_{0}, \pi\right) G_{w}\left(r, \theta ; r_{0}, \pi\right) d r_{0}, \\
& w^{(c-)}=-\int_{\Pi_{1}} \tau_{\theta z}^{(t)}\left(r_{0}, \pi\right) G_{w}\left(r, \theta ; r_{0}, \pi\right) d r_{0}-\int_{\Pi_{2}} \tau_{\theta z}^{(t)}\left(r_{0}, \pi\right) G_{w}\left(r, \theta ; r_{0}, \pi\right) d r_{0}, \\
& \text { where } \Gamma_{1} \in[C, C+d] ; \Gamma_{2} \in\left[C+d+A_{2}+2 * R_{2}, \infty\right] ; \Gamma_{3} \in\left[R_{1}, \infty\right] ; \\
& \Pi_{1} \in\left[R_{1}, C\right] ; \Pi_{2} \in\left[C+d, C+d+A_{2}\right] ; C=R_{1}+A_{1} .
\end{aligned}
$$

The integral equations to calculate the unknown force $f^{+}\left(r_{0}, \theta_{0}\right)$ can be obtained through conjunction of the semi-infinite parts as follows:

$$
\begin{aligned}
& \int_{\Gamma_{1}} f^{+}\left(r_{0}, \pi\right) G_{w}\left(r, \theta ; r_{0}, \pi\right) d r_{0}+\int_{\Gamma_{2}} f^{+}\left(r_{0}, \pi\right) G_{w}\left(r, \theta ; r_{0}, \pi\right) d r_{0}+\int_{\Gamma_{3}} f^{+}\left(r_{0}, 0\right) G_{w}\left(r, \theta ; r_{0}, 0\right) d r_{0} \\
& =-\int_{\Pi_{1}} \tau_{\theta z}^{(t)}\left(r_{0}, \pi\right) G_{w}\left(r, \theta ; r_{0}, \pi\right) d r_{0}-\int_{\Pi_{2}} \tau_{\theta z}^{(t)}\left(r_{0}, \pi\right) G_{w}\left(r, \theta ; r_{0}, \pi\right) d r_{0} \quad(\theta=0, \pi)
\end{aligned}
$$

The dimensionless DSIF at the tip of the right crack can be defined as:

$$
k_{3}^{\sigma}=\frac{1}{\tau_{0} Q}\left|\lim _{r_{0} \rightarrow R_{1}+A_{1}} f^{+}\left(r_{0}, \pi\right) \sqrt{2\left(r_{o}-R_{1}-A_{1}\right)}\right|, Q=\sqrt{R_{1}+A_{1} / 2}, \tau_{0}=c^{*} k w_{0} .
$$

\section{Numerical Example and Discussions}

In this section, some results for the DSIFs at the tip of the right crack are plotted to show the influence of the structural geometry, and the frequencies of the incident wave on the DSIFs.
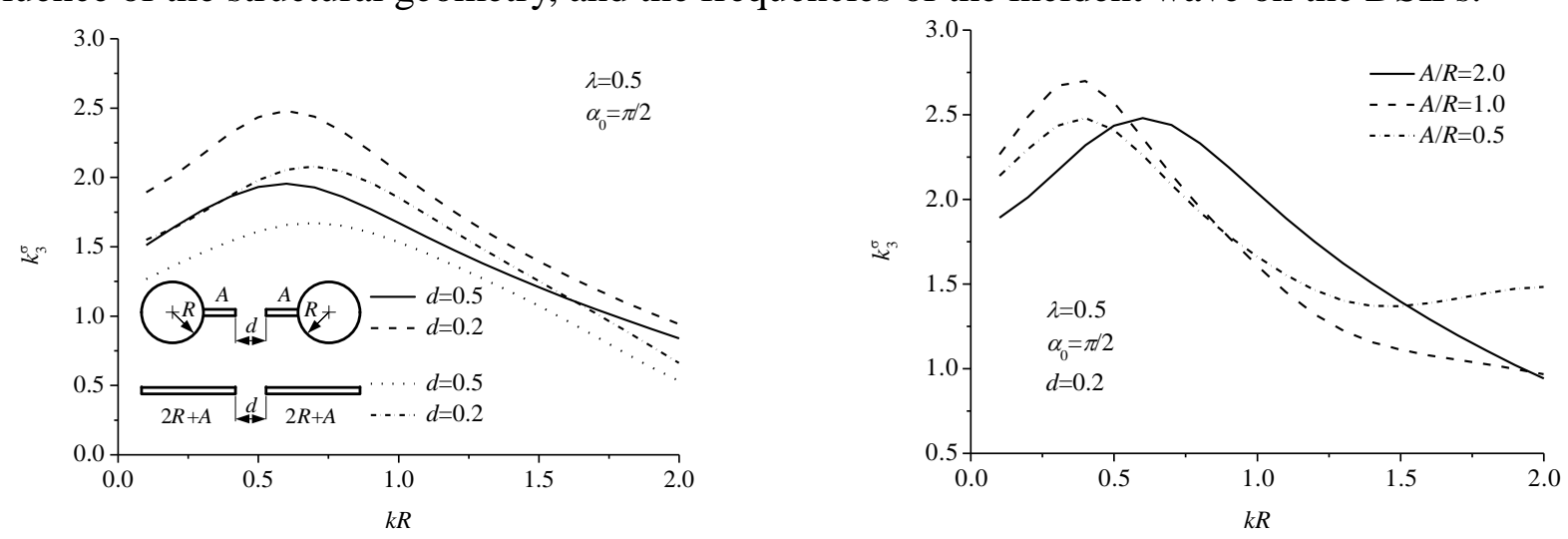

Fig. 3 DSIFs' comparison

Fig. 4 Variations of DSIFs with $k R$ for different $A / R$ 


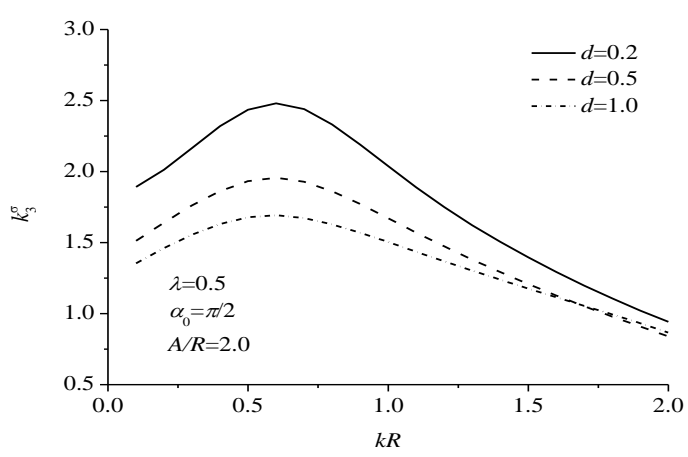

Fig. 5 Variations of DSIFs with $k R$ for different $d$

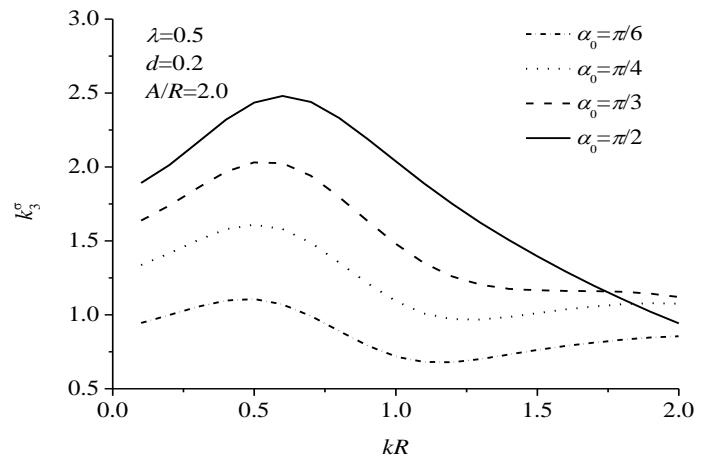

Fig. 6 Variations of DSIFs with $k R$ for different $\alpha_{0}$

First, a comparison of the DSIFs between two kinds of crack models is shown in Fig. 3. It can be noticed that DSIFs for the radical cracks on circular cavities are constantly larger than those for the straight-line cracks. And the curves of the straight-line crack model are also compared with the results of the document [1], it indicates that they coincide well.

At Fig. 4, the variation of the DSIFs with different $A / R$ is shown. It can be seen that DSIFs are larger at lower frequencies than those at higher frequencies, and the DSIFs is the largest at $A / R=1.0$, which indicates that the influence of the cavity on DSIF is more significant when the crack's lengths and the radii of the cavities are approximately equal.

Fig. 5 shows the variations of the DSIFs with different distance between the tips of the cracks. It is displayed that the values of the DSIFs increase with the decrease of the distance $d$. Fig. 6 implies that the DSIFs is the largest when the dynamic SH-wave is normally incident upon the piezoelectric material.

\section{Summary}

The comparison of the DSIFs between two kinds of crack models indicates that the values of radial crack-tip DSIFs on the circular cavities are larger then those on the reduced straight cracks with an effective length at the dynamic situation. The increase in the distance between the crack's tips decreases the DSIFs. The influence of the cavity on DSIF is more significant when the crack's lengths and the radii of the cavities are approximately equal. And the DSIFs are larger at lower frequencies than those at higher frequencies, so we must pay more attention to the fracture of engineering structures in low frequency. The method employed in present paper can serve as a useful reference for modeling of a piezoelectric material with complex defects and for the design of piezoelectric structures.

\section{Acknowledgement}

The present work is supported by the National Science Fund for Young Scholars of Hebei Education Department (Q2012031).

\section{References}

[1]X.D. Wang, S.A. Meguid, Modelling and analysis of the dynamic behavior of piezoelectric materials containing interacting cracks, Mechanics and Materials. 32 (2000) 723-737.

[2]X.D. Wang, On the dynamic behavior of interacting interfacial cracks in piezoelectric media, International Journal of Solids and Structures. 38 (2001) 815-831.

[3]D. Liu, Z. Chen, Scattering of SH-wave by cracks originating at an elliptic hole and dynamic stress intensity factor, Applied Mathematics and Mechanics. 25 (2004) 1047-1056. 
[4]J.H. Guo, Z.X. Lu, H.T. Han, Z. Yang, The behavior of two non-symmetrical permeable cracks emanating from an elliptical hole in a piezoelectric solid, Europe Journal of Mechanic A/Solids. 29 (2010) 654-663.

[5]A. Hassan, T.S. Song, Dynamic anti-plane analysis for two symmetrically interfacial cracks near circular cavity in piezoelectric bi-materials, Applied Mathematics and Mechanics. 35(10) (2014) 1261-1270. 